\title{
Correction to: Information Transmission and Criticality in the Contact Process
}

\author{
M. Cassandro ${ }^{1}$ - A. Galves ${ }^{2}$ • E. Löcherbach ${ }^{3}$
}

Published online: 29 November 2017

(C) Springer Science+Business Media, LLC, part of Springer Nature 2017

\section{Correction to: J Stat Phys (2017) 168:1180-1190 https://doi.org/10.1007/s10955-017-1854-3}

The original publication of the article unfortunately contained a mistake in the first sentence of Theorem 1 and in the second part of the proof of Theorem 1. The corrected statement of Theorem as well as the corrected proof are given below. The full text of the corrected version is available at http://arxiv.org/abs/1705.11150.

The first phrase of Theorem 1 is given as follows.

For any fixed $q>p>\frac{2}{3}$ there exist $\lambda_{c}<\lambda_{1}(p) \leq \lambda_{2}(p)$ such that the following holds. The corrected version of Step 2. of the proof of Theorem 1 is given as follows.

Proof of Theorem 1 Step 2 . We finally consider the case where $\lambda_{1}$ is sufficiently larger than $\lambda_{c}$ and where $q>p \geq \frac{2}{3}$. Let $Q$ be the monotone coupling between $\nu_{\lambda_{1}}$ and $\nu_{\lambda_{2}}$ induced by the construction of Proposition 2. Using this coupling and the fact that $f(0)=0$, we obtain thanks to Theorem 2 that

$$
\begin{aligned}
& \lim _{t \rightarrow \infty} \Delta_{p, q}\left(\lambda_{1}, \lambda_{2}, r, t\right) \\
& \quad=\varrho\left(\lambda_{2}\right) \int_{\mathcal{P}(\mathbb{Z})} f(|B \cap \Lambda|) v_{\lambda_{2}}(d B)-\varrho\left(\lambda_{1}\right) \int_{\mathcal{P}(\mathbb{Z})} f(|B \cap \Lambda|) \nu_{\lambda_{1}}(d B)
\end{aligned}
$$

The original article can be found online at https://doi.org/10.1007/s10955-017-1854-3.

$\bowtie$ E. Löcherbach

eva.loecherbach@u-cergy.fr

M. Cassandro

marzio.cassandro@gmail.com

\section{A. Galves}

galves@usp.br

1 Gran Sasso Science Institute, L'Aquila, Italy

2 Instituto de Matemática e Estatística, Universidade de São Paulo, São Paulo, Brazil

3 AGM, CNRS-UMR 8088, Université de Cergy-Pontoise, 95000 Cergy-Pontoise, France 


$$
\begin{aligned}
= & \varrho\left(\lambda_{1}\right) \iint\left(f\left(\left|B_{2} \cap \Lambda_{r}\right|\right)-f\left(\left|B_{1} \cap \Lambda_{r}\right|\right)\right) Q\left(d B_{1}, d B_{2}\right) \\
& +\left(\varrho\left(\lambda_{2}\right)-\varrho\left(\lambda_{1}\right)\right) \int_{\mathcal{P}(\mathbb{Z})} f(|B \cap \Lambda|) \nu_{\lambda_{2}}(d B) \\
= & : T_{1}(r)+T_{2}(r) .
\end{aligned}
$$

We want to show that this expression is negative for sufficiently large values of $\lambda_{1}$ and $r$. We put $\varepsilon:=2-p-q$. Since by assumption $q>p \geq \frac{2}{3}$, we have $2(1-\varepsilon)>\varepsilon$ (this will be important in (1.3) below).

Then $f(2)-f(1)=-(1-\varepsilon) f(1)$ and $f(2)=\varepsilon f(1)$. Writing for short

$$
Q(r, n, m):=Q\left(\left\{\left(B_{1}, B_{2}\right):\left|B_{1} \cap \Lambda_{r}\right|=n,\left|B_{2} \cap \Lambda_{r}\right|=m\right\}\right),
$$

it is clear that

$$
\begin{aligned}
T_{1}(r) & =\varrho\left(\lambda_{1}\right)[(f(2)-f(1)) Q(r, 1,2)+f(2) Q(r, 0,2)+f(1) Q(r, 0,1)] \\
& =\varrho\left(\lambda_{1}\right) f(1)[-(1-\varepsilon) Q(r, 1,2)+\varepsilon Q(r, 0,2)+Q(r, 0,1)] .
\end{aligned}
$$

Applying the last item of Theorem 2, we have that

$$
\begin{aligned}
\lim _{r \rightarrow \infty} T_{1}(r)= & \varrho\left(\lambda_{1}\right) f(1)\left(-2(1-\varepsilon)\left(\varrho\left(\lambda_{2}\right)-\varrho\left(\lambda_{1}\right)\right) \varrho\left(\lambda_{1}\right)\right. \\
& \left.+\varepsilon\left(\varrho\left(\lambda_{2}\right)-\varrho\left(\lambda_{1}\right)\right)^{2}+2\left(\varrho\left(\lambda_{2}\right)-\varrho\left(\lambda_{1}\right)\right)\left(1-\varrho\left(\lambda_{2}\right)\right)\right) \\
= & \varrho\left(\lambda_{1}\right) f(1)\left(\varrho\left(\lambda_{2}\right)-\varrho\left(\lambda_{1}\right)\right)\left(-2(1-\varepsilon) \varrho\left(\lambda_{1}\right)+\varepsilon\left(\varrho\left(\lambda_{2}\right)-\varrho\left(\lambda_{1}\right)\right)\right. \\
& \left.+2\left(1-\varrho\left(\lambda_{2}\right)\right)\right) .
\end{aligned}
$$

Moreover,

$$
\lim _{r \rightarrow \infty} T_{2}(r)=\left(\varrho\left(\lambda_{2}\right)-\varrho\left(\lambda_{1}\right)\right)\left[2 f(1) \varrho\left(\lambda_{2}\right)\left(1-\varrho\left(\lambda_{2}\right)\right)+f(2) \varrho\left(\lambda_{2}\right)^{2}\right] .
$$

Putting these results together, we conclude that

$$
\begin{aligned}
& \lim _{r \rightarrow \infty} \lim _{t \rightarrow \infty} \Delta_{p, q}\left(\lambda_{1}, \lambda_{2}, r, t\right)=\left(\varrho\left(\lambda_{2}\right)-\varrho\left(\lambda_{1}\right)\right) f(1) \\
& \quad \times\left\{-2(1-\varepsilon)\left(\varrho\left(\lambda_{1}\right)\right)^{2}+\varepsilon \varrho\left(\lambda_{1}\right)\left(\varrho\left(\lambda_{2}\right)-\varrho\left(\lambda_{1}\right)\right)+2\left(1-\varrho\left(\lambda_{2}\right)\right) \varrho\left(\lambda_{1}\right)\right. \\
& \left.\quad+2 \varrho\left(\lambda_{2}\right)\left(1-\varrho\left(\lambda_{2}\right)\right)+\varepsilon \varrho\left(\lambda_{2}\right)^{2}\right\} .
\end{aligned}
$$

Since $2(1-\varepsilon)>\varepsilon$, it is possible to choose $\delta^{*}$ such that for all $\delta \leq \delta^{*}$,

$$
2(1-\varepsilon)(1-\delta)^{2}-\varepsilon(1-\delta) \delta-4 \delta-\varepsilon(1-\delta)^{2} \geq \kappa>0,
$$

for some (sufficiently small) $\kappa>0$. Recall that $\varepsilon=\varepsilon(p)$. Since $\lim _{\lambda \uparrow \infty} \varrho(\lambda)=1$, we may choose $\lambda_{2}(p)$ sufficiently large such that $\varrho\left(\lambda_{1}\right) \geq 1-\delta^{*}$ for all $\lambda_{1} \geq \lambda_{2}(p)$. As a consequence, for all $\lambda_{2} \geq \lambda_{1} \geq \lambda_{2}(p)$,

$$
\lim _{r \rightarrow \infty} \lim _{t \rightarrow \infty} \Delta_{p, q}\left(\lambda_{1}, \lambda_{2}, r, t\right) \geq \kappa>0,
$$

which implies the assertion. 